\title{
El uso de cannabis aumentaría el riesgo de desarrollar un trastorno psicótico en el futuro
}

\author{
Cannabis use would increase the risk of future psychotic disorders
}

Moore y col. Lancet.2007; 370: 319-28.

\section{Objetivo}

Revisar sistemáticamente la evidencia científica acerca del uso de cannabis y la ocurrencia de episodios psicóticos o afectivos que perduran más allá de la intoxicación aguda.

\section{Fuente y selección de datos}

Los autores buscaron en las bases de datos Medline, Embase, CINAHL, PsycINFO, ISI Web of Knowledge, ISI Proceedings, ZETOC, BIOSIS, LILACS y MEDCARIB, desde su inicio hasta septiembre de 2006. De 4804 estudios encontrados se seleccionaron los 35 más homogéneos a criterio de los autores. Se incluyeron estudios poblacionales longitudinales y estudios caso-control longitudinales; se excluyeron cohortes de personas con enfermedad mental y/o problemas relacionados con el abuso de sustancias, estudios de población carcelaria y los estudios de uso medicinal de cannabis. Se consideró episodio psicótico al reporte de esquizofrenia, trastorno esquizofreniforme, trastorno esquizo-afectivo, psicosis no especificada, síntomas psicóticos, alucinaciones y desorden del pensamiento. Como episodio afectivo se incluyeron a los reportes de trastornos del humor, trastornos bipolares, trastorno del ánimo no especificado, depresión, ideación o intento suicida, ansiedad, neurosis y manía.

\section{Resultados principales}

Se encontró un riesgo incrementado de padecer algún tipo de episodio psicótico en los individuos que usaron cannabis (OR: 1,41 ; IC95\% 1,20 a 1,65). Los hallazgos fueron consistentes con un efecto dosis-respuesta, es decir que el riesgo más alto se vio en las personas que usaron cannabis en forma más frecuente (OR: 2.09; IC95\% 1,54 a 2,84).

La depresión, los pensamientos suicidas y la ansiedad, fueron examinados en forma separada. Los hallazgos para estos eventos fueron menos consistentes que para las psicosis.

\section{Conclusiones}

La evidencia es consistente con la apreciación de que el cannabis incrementa el riesgo de eventos psicóticos, independientemente de los efectos de la intoxicación aguda, siendo menos fuerte la evidencia respecto de los eventos afectivos. Si bien es poco probable poder responder si el cannabis es la causa de psicosis, los autores concluyen que hay suficiente evidencia como para advertir a los jóvenes que el uso de cannabis puede incrementar el riesgo de desarrollar una enfermedad psicótica a lo largo de la vida. Palabras clave: cannabis, marihuana, trastorno psicótico, riesgo.
Key words: cannabis, psychotic disorder, risk.

Fuente de financiamiento: agencias de salud estatales del RU.
Si el cannabis es el causante de un trastorno psicótico o si lo que hace es desenmascarar una psicosis latente es una vieja discusión entre los clínicos ${ }^{1,2}$. Hasta la fecha no hay investigaciones que afirmen una u otra cosa. De hecho, el presente trabajo tampoco lo hace pero sí aporta algo muy valioso: haber corroborado en población sana al inicio del estudio -por lo menos sin manifestaciones clínicas de las enfermedades estudiadas- y con seguimientos a largo plazo, que a mayor consumo de cannabis, existe mayor riesgo de presentar un evento psicótico a lo largo de la vida y especialmente, que este evento puede producirse alejado del momento de consumo, momento en el que es esperable algún tipo de sintomatología psiquiátrica.

Vale aclarar que los hallazgos de la revisión sistemática de Moore y col. son compatibles con varios de los criterios postulados por Bradford Hill hace más de cuarenta años para establecer causalidad ${ }^{3}$ : fuerza de la asociación, consistencia, secuencia temporal (que la causa anteceda al efecto) gradiente biológico (efecto dosis-respuesta) plausibilidad biológica y coherencia entre diferentes investigaciones.

Tan importante es este hallazgo como históricamente lo fue el encontrar que el uso de tabaco aumenta el riesgo desarrollar cáncer de pulmón o enfermedad cardiovascular, comparado con quien nunca probó un cigarrillo 4 .

\section{Conclusión del comentador}

La evidencia va contradiciendo cada vez más a quienes aseguran que el cannabis no es nocivo y que por ello debería despenalizarse su uso. Sería bueno que trabajos como este fueran más difundidos entre la comunidad médica ya que mal puede aconsejar, quien no esta convencido de lo que aconseja. No olvidemos que la puesta en práctica en forma masiva del consejo antitabaco llegó de la mano de la evidencia que los trabajos de investigación fueron aportando, hasta que no hubo forma de dudar de su lineal relación con las enfermedades ya nombradas.

David Orozco [ Especialista en Psiquiatría. Director Médico de CETRAD, Programa Despertares, Institución especializada en el tratamiento de las adicciones. davidorozco@flashmail.com ]

Orozco D. El uso de cannabis aumenta el riesgo de desarrollar un trastorno psicótico. Evid. actual. práct. ambul; 10(5): 145, Sep-Oct.2007. Comentado de: Moore T y col. Cannabis use and risk of psychotic or affective mental health outcomes: a systematic review. 2007 Jul 28; 370 (9584):31928. PMID: 17662880. Disponible en URL: http://www.t-act.info/Moore-Lancet-MJPsychosis.pdf

Referencias

1. Henquet C, Murria R, Linszen D, Van Os J. Ambiente y esquizofrenia: el papel del consumo de cannabis. Schizophrenia Bulletin. 2005; 31(3)

2. Roncero C, Collazos F, Valero S, Casas M. Consumo de cannabis y desarrollo de psicosis: estado actual. Actas Españolas de Psiquiatría. 2007 May;35(3).

3. Austin Bradford Hill, "The Environment and Disease: Association or Causation?" Proceedings of the Royal Society of Medicine, 58 (1965), 295-300. Disponible en URL: http://www.edwardtufte.com/tufte/hill

4. López A. Medición de los riesgos del tabaco para la salud: un comentario. Bulletin of the World Health Organization, 1999, 77 (1): $82 \pm 83$. Disponible en URL: http://whqlibdoc.who.int/boletin/1999/RA_1999_1_180-181_spa.pdf 